\title{
Simulations of Dual-Vortex Interaction within Environmental Shear
}

\author{
Robert E. Hart And Jenni L. Evans \\ Department of Meteorology, The Pennsylvania State University, University Park, Pennsylvania
}

(Manuscript received 28 February 1997, in final form 25 January 1999)

\begin{abstract}
For over a century it has been known that each vortex in a multiple vortex configuration will move in response to the other vortices. However, despite advances since that time, the complexities of multiple vortex scenarios when sheared environments are present are still not completely understood. The interaction of binary vortices within horizontal environmental shear is explored here through shallow water simulations on a $\beta$ plane. Due to nonlinear feedbacks, the combination of environmental vorticity (or vorticity gradient) and shear, as well as the multiple vortex situation, results in a more complicated track than for a storm experiencing any individual component. Despite the complexity of these vortex-environment interactions, the use of previous single-vortex studies greatly aids interpretation. Centroid-relative motion of the individual vortices is considered, as well as the propagation of the vortex pair centroid, to understand motion effects of the different vortex-environment combinations.

As the vortices interact, vortex Rossby waves are generated through distortion of the symmetric vorticity field by the opposing vortex. Initially, the high-frequency waves have an insignificant effect upon vortex intensity or propagation, and $\beta$-induced wavenumber one asymmetry dominates as expected. However, as the waves approach a critical radius $(\zeta=0)$, wave potential vorticity filamentation and stretching by the circulation of the adjacent vortex leads to a coupling of the two vortices. This vortex coupling results in enhanced propagation speeds of the two vortices proportional to the effective size of the dual-vortex system.

The sign of vorticity of the environmental flow can act to enhance or negate $\beta$-drift such that single- or dualvortex propagation is altered. Further, when environmental vorticity is present, the rate of mutual orbit from Fujiwhara rotation is altered. When the environmental flow is cyclonic, the cyclonic mutual rotation of the vortices is accelerated. Conversely, when the environmental flow is anticyclonic, the mutual rotation of the vortices is substantially decelerated, but remains cyclonic.
\end{abstract}

\section{Introduction}

For over a century it has been known that each vortex in a multiple vortex configuration will move in response to the other vortices (Helmholtz 1867; Kirchoff 1876; Fujiwhara 1921). Dynamically, this motion results from the mutual advection of the symmetric vorticity associated with each vortex. More recently, Adem (1956) and many others have documented the contribution to vortex motion resulting from vortex interaction with the earth's background vorticity gradient, the $\beta$ effect. The earth's vorticity gradient is analogous here to the vorticity field associated with an additional vortex of infinite scale. In a system of interacting barotropic vortices, each vortex will respond to the environmental vorticity gradient provided by the presence of the others (DeMaria and Chan 1984). Hence, a vortex will be both advected by its partner and will propagate on the environmental vorticity gradient resulting from both $\beta$ and

Corresponding author's address: Mr. Robert E. Hart, 503 Walker Bldg., Department of Meteorology, The Pennsylvania State University, University Park, PA 16802.

E-mail: hart@ems.psu.edu the partner vortex (Holland and Dietachmayer 1993). Idealized studies of (single vortex) tropical cyclone motion in spatially varying environments have generally characterized the storm environment as a shear flow representative of observed ridge and trough features (e.g., Evans et al. 1991; Williams and Chan 1994; Chan and Law 1995). The combination of all of these effects: $\beta$-induced propagation, vortex-induced advection and propagation, as well as environmental flows, will be investigated in the present study.

Observational studies of multiple vortex interactions demonstrate occurrence of binary interactions in virtually all years for the western North Pacific and about every 3 yr for the North Atlantic (e.g., Brand 1970; Dong and Neuman 1983). The approach of two tropical cyclones within a critical separation distance would result in a binary storm interaction (Brand 1970; Dong and Neuman 1983; Lander and Holland 1993). Brand (1970) theorized that the critical separation distance would be proportional to the tropical cyclone sizes; however, it is only recently that data capable of providing size climatologies have become available (e.g., Liu and Chan 1997).

In the study presented here, progressively more com- 
plex environmental flows are overlaid on a pair of identical tropical cyclonelike vortices, initially located 500 $\mathrm{km}(5 \times$ radius of maximum winds $)$ apart on a $\beta$ plane centered on $20^{\circ} \mathrm{N}$. The entire system is barotropic and there is no meridional flow in the initial background wind field. Justification for the continued use of barotropic dynamics is drawn from a number of studies of tropical cyclonelike vortices (e.g., Chang 1982, 1984; Holland and Dietachmayer 1993), which demonstrate that the phases of Fujiwhara interaction are well simulated by barotropic physics alone.

\section{Methodology}

A shallow water equations model is employed here to investigate the barotropic response of a pair of cyclonic vortices embedded in a sequence of prescribed zonal shear flows. The model is reviewed briefly here. The prescribed initial conditions are summarized in section $2 \mathrm{~b}$ and Table 1.

\section{a. Model summary}

The shallow water equations model is based on that used by Evans et al. (1991). Temporal differencing is modified Euler-backward. Arakawa and Lamb's (1981) enstrophy conserving spatial differencing is used on a $20-\mathrm{km}$ grid. The domain measures between 5000 and $8000 \mathrm{~km}$ on a side (depending on the experiment) and is centered on a $\beta$ plane at $20^{\circ} \mathrm{N}$. The equivalent depth is set at $5000 \mathrm{~m}$ and so the results obtained here are essentially nondivergent. The boundaries of the domain form a channel with inviscid walls to the north and south, and cyclic lateral boundaries.

As discussed by Chan and Williams (1987), a single vortex on a $\beta$ plane can distort over time in a manner analogous to Rossby wave dispersion. Such dispersive effects limit the useful time of integration for the cases studied here. Hence, although all cases are run for well over $200 \mathrm{~h}$ (and often as much as $420 \mathrm{~h}$ ), inspection of the time-evolving potential vorticity (PV) fields reveals that the results are likely modulated by dispersive waves reentering the eastern boundary after 7 days (168 h). Discussion of the vortex evolution will therefore be confined to the first 7 days of each integration.

\section{b. Initial conditions}

Six case studies, forming a hierarchy of environment flow complexity, will be described here. In all cases, the initial vortices are identical to each other in all respects and are located $500 \mathrm{~km}$ apart at the same latitude. The prescribed background flows are purely zonal $\mathbf{v}=$ $u(y) \mathbf{i}(y=$ latitudinal displacement in $\mathrm{km}$ ) and each of the prescribed environmental flows are in geostrophic balance. The structure of these sheared environments is chosen to control potential vorticity characteristics. Balance is attained in the initial conditions (environmental

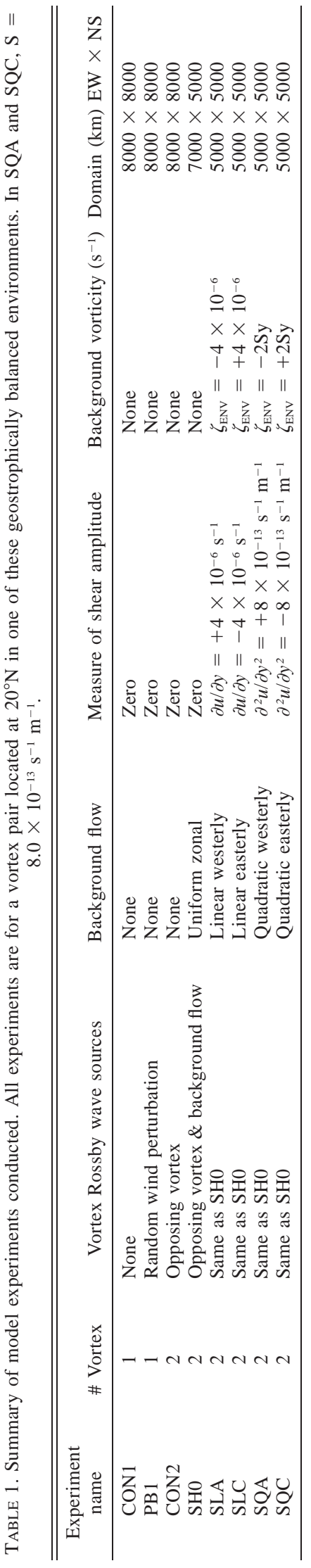


flow with both vortices) via dynamic initialization (Satomura 1980).

\section{1) Vortex STRUCTURE AND A CONTROL EXPERIMENT, CON2}

Two modified Rankine vortices are placed in a quiescent environment on a $\beta$ plane at $20^{\circ} \mathrm{N}$. The radial $(r)$ dependence of the vortex tangential wind is prescribed according to

$$
v=\sqrt{\left(\frac{b r_{\max } h_{C}}{r^{b}} \exp \left[-\left(\frac{r_{\max }}{r}\right)^{b}\right]+\frac{r^{2} f^{2}}{4}\right)}-\frac{r f}{2},
$$

where $f$ is the Coriolis parameter, $r_{\max }=100 \mathrm{~km}$ is the location of the maximum tangential wind and $h_{c}$ (vortex height perturbation) is chosen so that $v_{\max }=25 \mathrm{~m} \mathrm{~s}^{-1}$. The factor $b=1.5$ determines the shape of the vortex outer wind structure and is chosen so that the cyclonic vorticity associated with the vortex is confined within $300-\mathrm{km}$ radius. Radial profiles of the vortex rotational winds, vorticity, and height of the free surface are plotted in Fig. 1. The model domain in this simulation is $8000 \mathrm{~km} \times 8000 \mathrm{~km}$. A single simulation with the same configuration, but on an $f$ plane, was also done for reference. All further simulations are for a $\beta$ plane.

\section{2) Two VORTICES IN AN ENVIRONMENT WITH NO SHEAR, SHO}

A uniform zonal flow of $5 \mathrm{~m} \mathrm{~s}^{-1}$ is superimposed on the initial conditions for the control experiment. The width of the model domain is $7000 \mathrm{~km}$. For the environmental flow to be in geostrophic balance, the meridional structure of the height field has the form

$$
h=\frac{5 y}{g}\left(f_{0}+\frac{\beta y}{2}\right),
$$

where $f_{0}$ is Coriolis parameter at $\mathrm{y}=0$. Meridional variation of this height field (dotted) is plotted in Fig. 2a, along with the corresponding zonal flow (solid), vorticity (dot-dash), and PV (dashed).

\section{3) TWO VORTICES IN ANTICYCLONIC, LINEAR SHEAR, SLA}

The vortices are situated in a geostrophic, zonal flow with anticyclonic, linear shear. The magnitude of the shear is $+4 \times 10^{-6} \mathrm{~s}^{-1}\left(0.8 f_{0}\right)$, with strongest easterlies $\left(-10 \mathrm{~m} \mathrm{~s}^{-1}\right)$ in the model Tropics and the strongest westerly flow at higher latitudes $\left(+10 \mathrm{~m} \mathrm{~s}^{-1}\right.$ ) (see Fig. $2 b)$. This configuration corresponds to a vortex pair located at $y=2000 \mathrm{~km}$, due south of a ridge axis at 2500 $\mathrm{km}$.

\section{4) CyClONIC, LINEAR SHEAR EXPERIMENT, SLC}

The vortex environment now consists of geostrophic, zonal flow with cyclonic, linear shear. The shear has a magnitude of $-4 \times 10^{-6} \mathrm{~s}^{-1}$. Meridional variation of zonal flow (solid), height (dotted), and PV (dashed) is plotted in Fig. 2c. A trough axis lies along $y=2500$ $\mathrm{km}$.

\section{5) ANTICYCLONIC, QUADRATIC SHEAR EXPERIMENT, SQA}

In all of the previous experiments, the relative vorticity of the environmental flow is constant. In this and the final experiment, the vortex pair is placed in a geostrophic, zonal flow with quadratic shear. For this experiment the shear is anticyclonic and the magnitude of the shear gradient, $S$, is $+8 \times 10^{-13} \mathrm{~s}^{-1} \mathrm{~m}^{-1}(0.04 \beta)$. The background zonal flow is

$$
U_{0}=S y^{2}-5 \text {. }
$$

The corresponding meridional variation of all environment quantities is indicated in Fig. $2 \mathrm{~d}$.

\section{6) CYCLONIC, QUADRATIC SHEAR EXPERIMENT, SQC}

This last experiment completes this survey, with the vortex pair situated in a cyclonic environmental flow with quadratically varying zonal winds (shown graphically in Fig. 2e):

$$
U_{0}=-S y^{2}+5 .
$$

Two additional sets of experiments were performed to isolate the potential for vortex Rossby wave generation due to barotropic instability. These are purely sensitivity analyses and do not form part of the vortexenvironment sequence, but are briefly described here for ease of reference.

\section{7) CONTROL VORTEX IN QUIESCENT ENVIRONMENT ON AN $f$ PLANE, CON1}

No vortex perturbation is introduced in this experiment. This forms the simplest control simulation for the system.

\section{8) BAROTROPICALLY STABLE VORTEX IN QUIESCENT ENVIRONMENT, PB1}

Two experiments fall under the banner of PB1. The same barotropically stable vortex is used in a quiescent environment for an $f$-plane simulation and a separate $\beta$-plane simulation. In each case, a perturbation of 10 $\mathrm{m} \mathrm{s}^{-1}$ was introduced at the radius of maximum winds at the initial time only. 


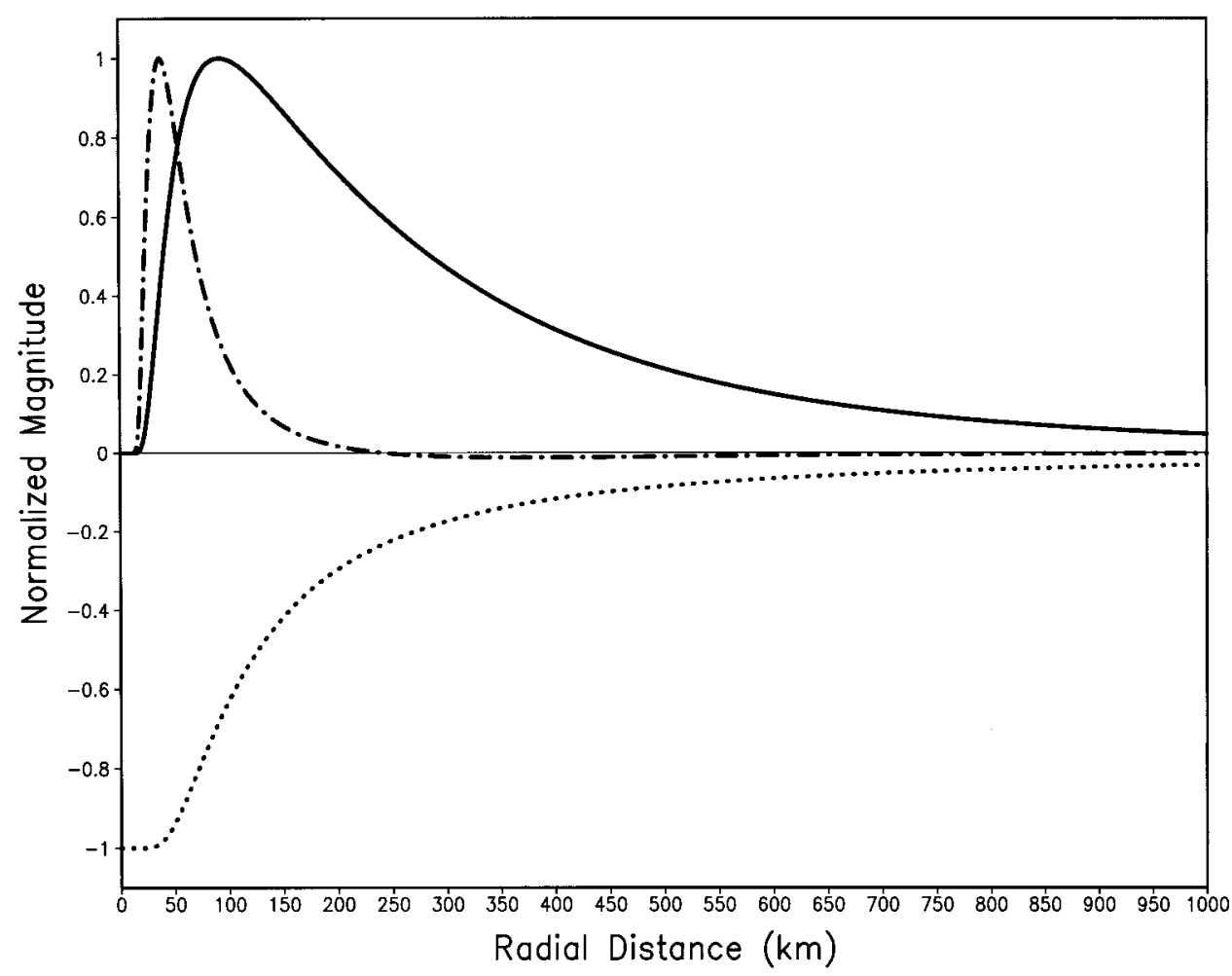

FIG. 1. Normalized radial profiles of the initial (modified Rankine) vortices. Maximum tangential winds (solid) of $25 \mathrm{~m} \mathrm{~s}^{-1}$ are initially located at RMW $=100 \mathrm{~km}$ (the radius of maximum winds), with maximum vertical vorticity (dot-dash) around $50 \mathrm{~km}$. The vorticity becomes anticyclonic at approximately $250 \mathrm{~km}$, reaching a minimum near $400 \mathrm{~km}$. The maximum height perturbation (dotted) is $115 \mathrm{~m}$; maximum vorticity is $2.8 \times 10^{-4} \mathrm{~s}^{-1}$. For comparison, the Coriolis parameter at $20^{\circ} \mathrm{N}$ is $5.5 \times 10^{-5} \mathrm{~s}^{-1}$. Environmental vorticity in each of the shear experiments has amplitudes: SH0 $=$ zero; SLA $=-4 \times 10^{-6} \mathrm{~s}^{-1}$; SLC $=+4 \times 10^{-6}$ $\mathrm{s}^{-1}$; SQA ranges from zero at minimum $\left(y=y_{\min }\right.$ to $-8 \times 10^{-6} \mathrm{~s}^{-1}$ at maximum $\left(y=y_{\max }\right)$; and SQC ranges from zero at minimum $\left(y=y_{\min }\right.$ to $+8 \times 10^{-6} \mathrm{~s}^{-1}$ at maximum $\left(y=y_{\max }\right)$.

\section{Vortex Rossby wave generation and filamentation}

Vortex-vortex interactions at very early stages of the no-shear experiment, SH0, are illustrated in Fig. 3 (SH0 PV field). High (more cyclonic) PV is shaded darker that less cyclonic flow, and white is essentially the zero $\mathrm{PV}$ region, bounding PV values $\pm 4 \times 10^{-9} \mathrm{~s}^{-1} \mathrm{~m}^{-1}$. Four time frames $(t=0,2 \mathrm{~h}, 4 \mathrm{~h}$, and $6 \mathrm{~h})$ early in the simulation are indicated. This immediate interaction of the two vortices is observed in each case reviewed here. Further experiments (PB1, discussed below) rule out barotropic instability of the vortices themselves as the source of these PV waves. Potential for any initial numerical imbalances has been minimized, since the vortex-environment system has been subjected to a dynamic initialization.

The vortices begin to rotate cyclonically about their centroid almost immediately ( $2 \mathrm{~h})$ and distortion of the vortex PV is evident by $4 \mathrm{~h}$. At $6 \mathrm{~h}$ this distortion is clearly identified as waves in the PV field, intruding as close as $200 \mathrm{~km}$ away from the individual vortex center. The regions in which these waves have largest ampli- tudes are collocated with regions of maximum convergence. Figure 4a shows the propagation of wave packets (kinetic energy maxima associated with the waves) in raduis-time coordinates; from this figure, we find the group velocities of $5 \mathrm{~m} \mathrm{~s}^{-1}$. Figure $4 \mathrm{~b}$ plots the asymmetric wind and PV field associated with the waves. From Fig. $4 \mathrm{~b}$, the phase speeds of the waves are of magnitude 5-10 $\mathrm{m} \mathrm{s}^{-1}$ with wavelengths of $25-50 \mathrm{~km}$. The phase speeds and group velocities of these waves are similar to the vortex Rossby waves simulated by Montgomery and Kallenbach (1997) in their shallowfluid model. From this we conclude that the waves generated here are vortex Rossby waves.

To isolate the source of the vortex Rossby waves, we performed an additional set of experiments involving only one vortex in a quiescent environment (CON1 and PB1). Through experiments with other vortex profiles (not shown), we have found that these waves occur regardless of whether the vortex profile is barotropically unstable, eliminating that instability as the source of the waves. Further, the waves are more easily studied if the vortex is not moving from $\beta$ or a mean flow, such that 

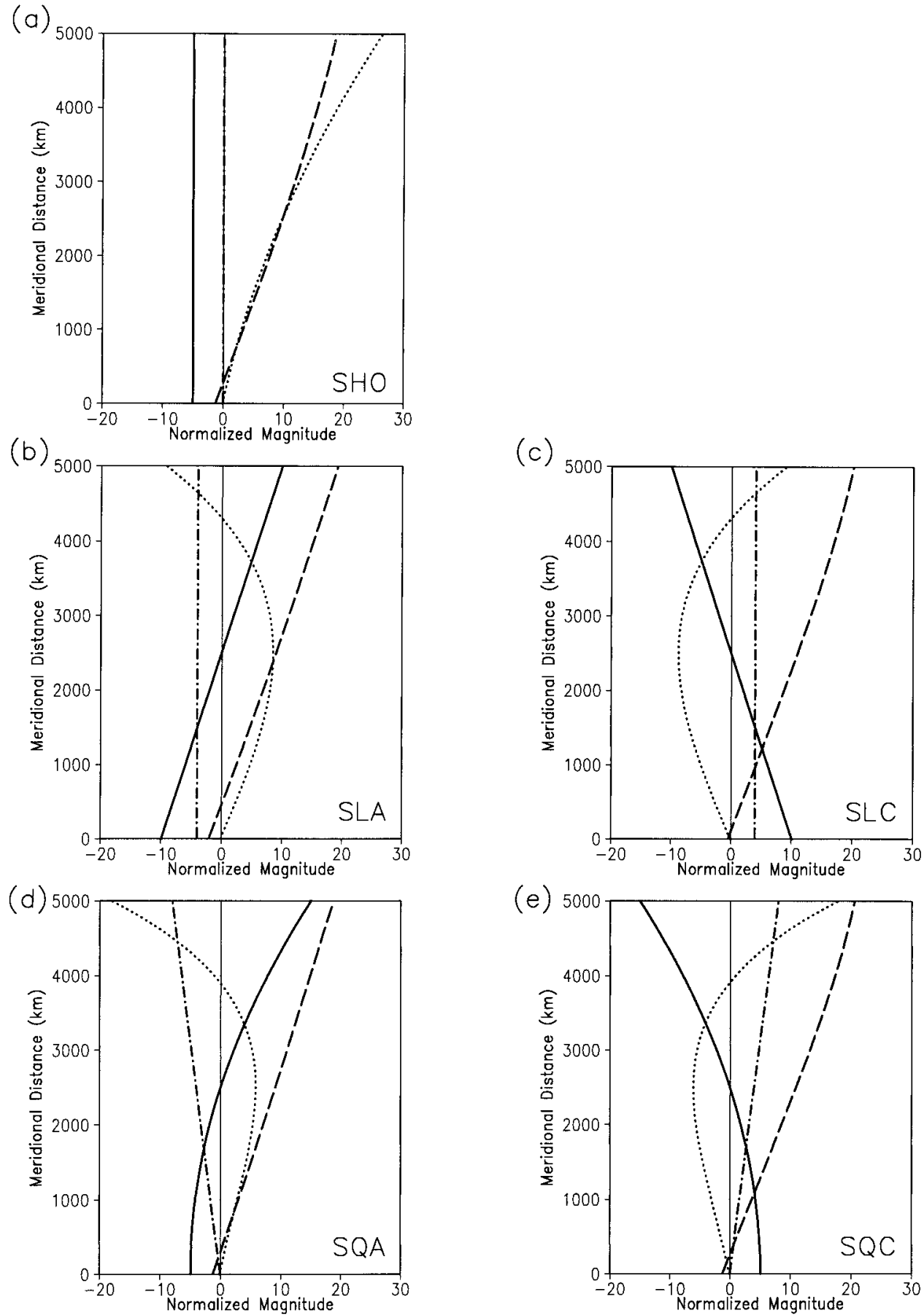

FIG. 2. Meridional profiles of environmental wind ( $\mathrm{m} \mathrm{s}^{-1}$; solid), perturbation height (dm; dotted), relative vorticity $\left(10^{-5} \mathrm{~s}^{-1}\right.$; dot-dashed), and PV $\left(10^{-8} \mathrm{~s}^{-1} \mathrm{~m}^{-1}\right.$; dashed) for shear experiments (a) SH0, (b) SLA, (c) SLC, (d) SQA, and (e) SQC, respectively. The wind and height fields are in geostrophic balance.

a wavenumber one asymmetry does not develop. However, we still needed to provide a source for the vortex Rossby waves since the natural perturbations are removed through these restrictions. Thus, we performed a subset of experiments where a single vortex is per- turbed with a random wind perturbation on an $f$ plane (PB1). The vortex Rossby waves are produced from this single, instantaneous wind perturbation and the waves have very similar structure to those in the SHO experiment discussed above. This set of experiments confirms 

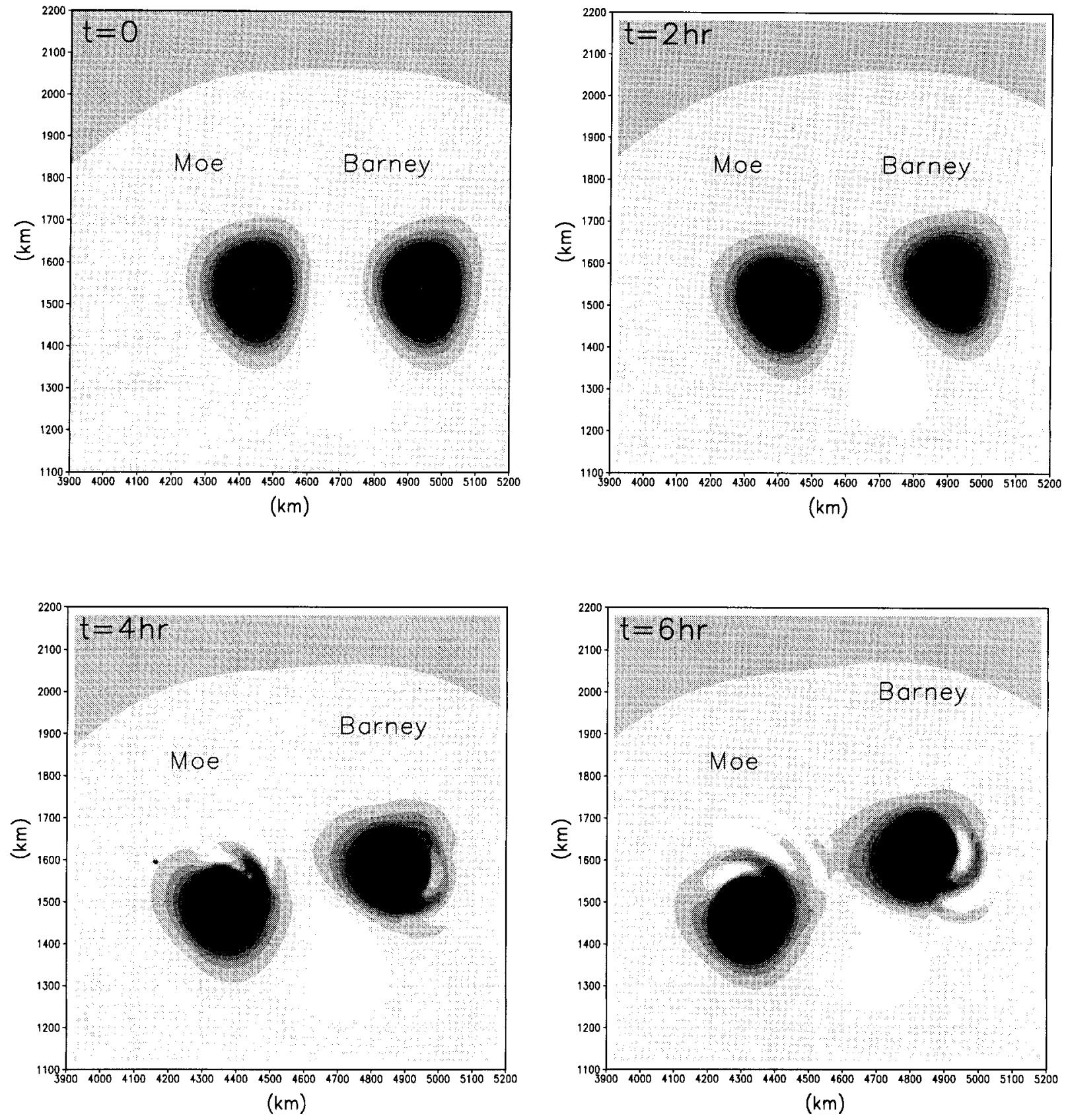

FIG. 3. Potential vorticity plots during the first $6 \mathrm{~h}$ of SH0: (a) $0 \mathrm{~h}$, (b) $2 \mathrm{~h}$, (c) $4 \mathrm{~h}$, and (d) $6 \mathrm{~h}$. The domain in all cases is $1300 \mathrm{~km} \times$ $1100 \mathrm{~km}$, much smaller than the total model domain. Maximum PV values are represented by black shading $\left(\mathrm{PV}>1.2 \times 10^{-7} \mathrm{~m}^{-1} \mathrm{~s}^{-1}\right)$; near-zero PV regions are white.

Montgomery and Kallenbach (1997) that the vortex Rossby waves are produced from distortion of the vortices' symmetric PV field.

The structure of the vortex Rossby waves changes with time. The asymmetric component of the wind field at $5,10,15$, and $20 \mathrm{~h}$ of integration for PB1 is plotted in Fig. 4b. Formation and propagation of the vortex Rossby waves are clearly shown. The azimuthal and radial wavenumber components of the waves increase with time. Shapiro and Montgomery (1993) found that the two-dimensional wavenumber of the vortex Rossby waves on an $f$ plane follow the relation:

$$
\frac{D k}{D t}=-n \frac{\partial}{\partial r}\left(\frac{\bar{v}}{r}\right),
$$

where $k$ is the radial wavenumber, $n$ is the azimuthal wavenumber, and the derivative is a measure of the shear 
(a)

(b)
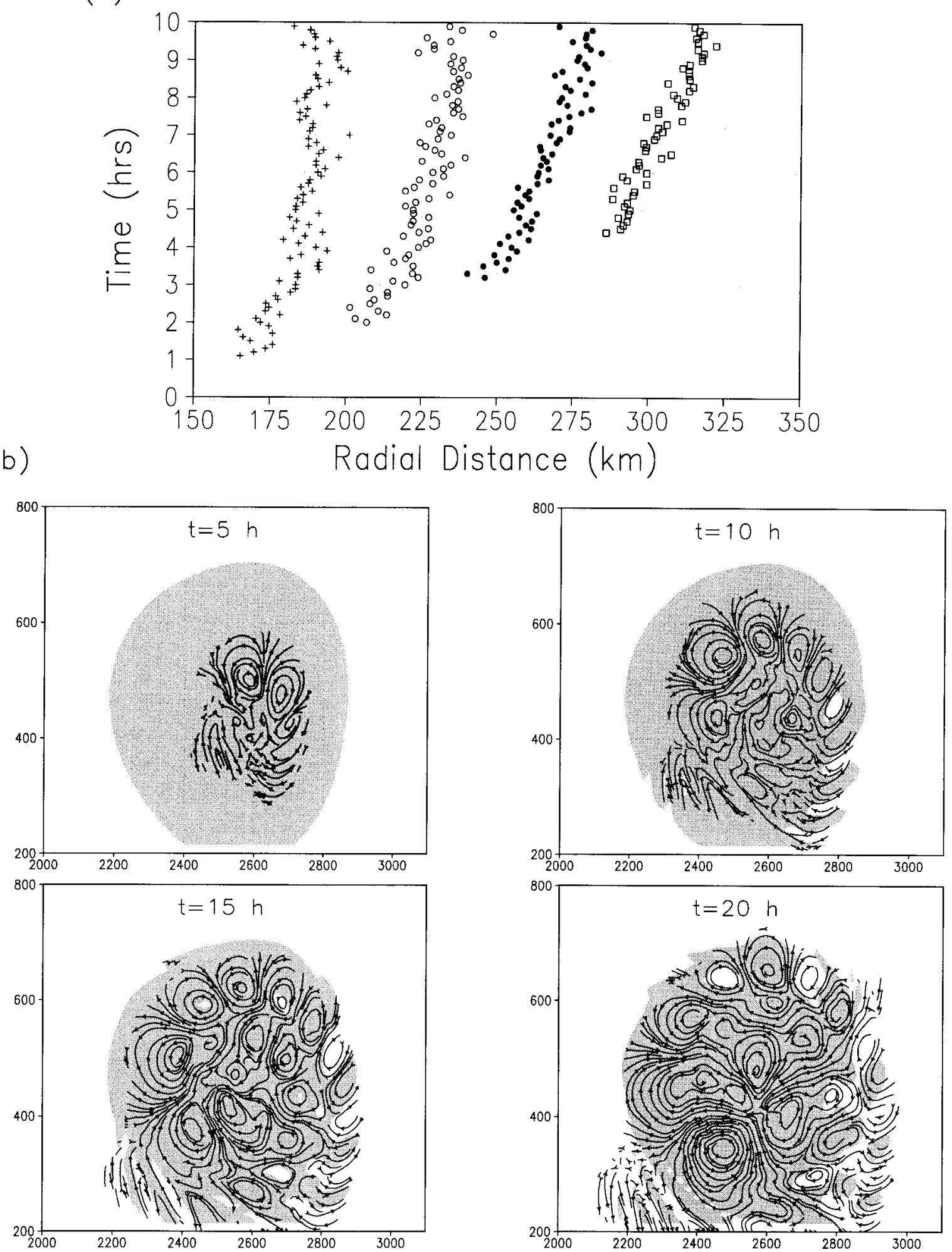

FIG. 4. Analyses of the vortex Rossby waves during the PB1 experiment when a random wind perturbation was applied at the radius of maximum winds: (a) propagation of the vortex Rossby wave packets as a function of radius and time in the perturbed PB1 experiment; (b) $1 \times 10^{-8} \mathrm{~m}^{-1} \mathrm{~s}^{-1} \mathrm{PV}$ field (shaded) and asymmetric wind field at 5,10,15, and $20 \mathrm{~h}$ when the vortex initial base state is removed. 
of the vortex wind profile. The wave evolution in Fig. $4 \mathrm{~b}$ qualitatively conforms to this relationship. As time increases, the radial wavenumber increases and the wave generation is greatest where the vortex shear is greatest (near the radius of maximum winds). Since only the radial wavenumber component increases with time under linear dynamics (5), the observed azimuthal component increase shown in Fig. $4 \mathrm{~b}$ must be attributable to nonlinearities resulting from wave-wave interactions.

On an $f$ plane, the waves clearly introduce a higherfrequency asymmetry into the circulation (Fig. 4b). The amplitude of this asymmetry is sufficiently small that the evolution of the wavenumber one asymmetry and hence vortex propagation and intensity is not significantly altered when on a $\beta$ plane. At 75 h on a $\beta$ plane, the track of the perturbed vortex (PB1, not shown) departs less than $20 \mathrm{~km}$ (one grid point) from the unperturbed vortex track (CON1, not shown). Further, the vortex intensity in the perturbed run is within $2 \%$ of the intensity in the unperturbed run. As a result, we find that the vortex Rossby waves are merely incidental during the early stages of vortex interaction. Initially, the waves are simply a convenient indicator that the vortex vorticity field is being distorted by an external source.

Beyond $75 \mathrm{~h}$, however, the vortex Rossby waves approach the critical radius of $350 \mathrm{~km}$, where the vortex relative vorticity becomes anticyclonic. The waves were observed to stagnate at this radius. This radius represents a limiting radius beyond which the vortex Rossby waves are unable to propagate (analogous to critical latitudes on earth). The PV associated with the wave filaments as it approaches the critical radius and is stretched azimuthally around the vortex. During these latter stages of interaction, we argue that the waves become more than incidental. The impact of this filamentation on the vortex circulation and propagation in dualvortex experiments is discussed next.

\section{Environmental influence on track and propagation}

Each of the cases outlined here is reviewed in terms of their tracks and intensity change to investigate vortex-vortex interaction in more detail. The actual and centroid-relative tracks for each case are plotted (Figs. 5 and 8). Since the initial vortices have identical size, structure, and intensity (retaining this form throughout the integration), the centroid is simply the arithmetic mean location of their centers.

Tracks for the binary vortex system in a quiescent environment (CON2) are shown in Fig. 5: (a) actual tracks, plus the centroid of the two vortices, (b) the centroid-relative motion, and (c) the centroid-relative motion of the experiment with the same two vortices on an $f$ plane. Introduction of a varying Coriolis parameter corresponds to the appearance of an abrupt track change in the $\beta$-plane study (Fig. 5b) that is not present for the same system on an $f$ plane (Fig. 5c). Indeed, without any background variation (and no radial flow associated with the vortices), the two vortices on an $f$ plane simply orbit cyclonically apart for as long as the simulation is run (Fig. 5c).

The tracks of the two vortices in this control $\beta$-plane experiment (CON2) seem very complex (Fig. 5a), in spite of the lack of any environmental forcing. In fact, the tracks of all of the nonzero environment systems (Fig. 8) appear to be more straightforward. The motion of the centroid in CON2 is steadily northwestward for the first $120 \mathrm{~h}$, turning more northward at that time. The centroid motion is consistent with $\beta$-induced (predominantly westward) propagation of $2.4 \mathrm{~m} \mathrm{~s}^{-1}$ until $96 \mathrm{~h}$, reducing to $1.75 \mathrm{~m} \mathrm{~s}^{-1}$ after the northward turn. As they approach the turn (i.e., northward motion of the centroid), the vortices have begun to move further apart (Fig. 6). Prior to this, the vortices had initially rotated cyclonically about the centroid for the first $72 \mathrm{~h}$, slowing their rotation rate between 72 and $120 \mathrm{~h}$ and then commencing anticyclonic rotation until $168 \mathrm{~h}$ (and beyond; Fig. 5b).

Inspection of the CON2 height field at 24-h intervals (Fig. 6) and the PV field (not shown) confirms that the two vortices rotate cyclonically in the first $48 \mathrm{~h}$ and then remain oriented north-south for the next $48 \mathrm{~h}$ as the entire system propagates to the northwest. The vortex separation distance is constant over the $48 \mathrm{~h}$ for which the north-south orientation is maintained, but increases after $96 \mathrm{~h}$, when anticyclonic rotation commences (Fig. 7).

One plausible explanation for the track-separation variations is as follows: the two vortices rotate in the first $48 \mathrm{~h}$ as two interacting systems, all the while modifying the outer vortex structure of each other; by 48 $\mathrm{h}$, a self-sustaining, coupled system has developed with the vortices maintaining a north-south orientation and moving as one, larger system. The coupling cannot be maintained by $96 \mathrm{~h}$, due to the evolution of an anticyclonic $\beta$ gyre between them, and the vortices proceed to move apart, operating as distinct units again. The slowing of the centroid propagation (from $2.4 \mathrm{~m} \mathrm{~s}^{-1}$ to $96 \mathrm{~h}$, then $1.75 \mathrm{~m} \mathrm{~s}^{-1}$ ) is consistent with the vortices propagating as a coupled, larger system until $96 \mathrm{~h}$ and as two distinct, smaller systems at later times. This agrees with a previous study that showed the propagation speed of vortex generally increases as the vortex size increases (Fiorino and Elsberry 1989). The track evolutions for the remaining cases will be briefly reviewed here and then this hypothesis for track transitions will be developed further in the next section.

The observed and centroid-relative tracks for the two vortices in a constant environmental flow (SH0) are plotted in Fig. 8a. The vortices mean zonal motion is dominated by the advection, with translation speed of $5.1 \mathrm{~m} \mathrm{~s}^{-1}$. Cyclonic rotation of the two vortices about their center is evident for the first $96 \mathrm{~h}$, with an abrupt and sustained change to anticyclonic rotation after this time. 
(a)

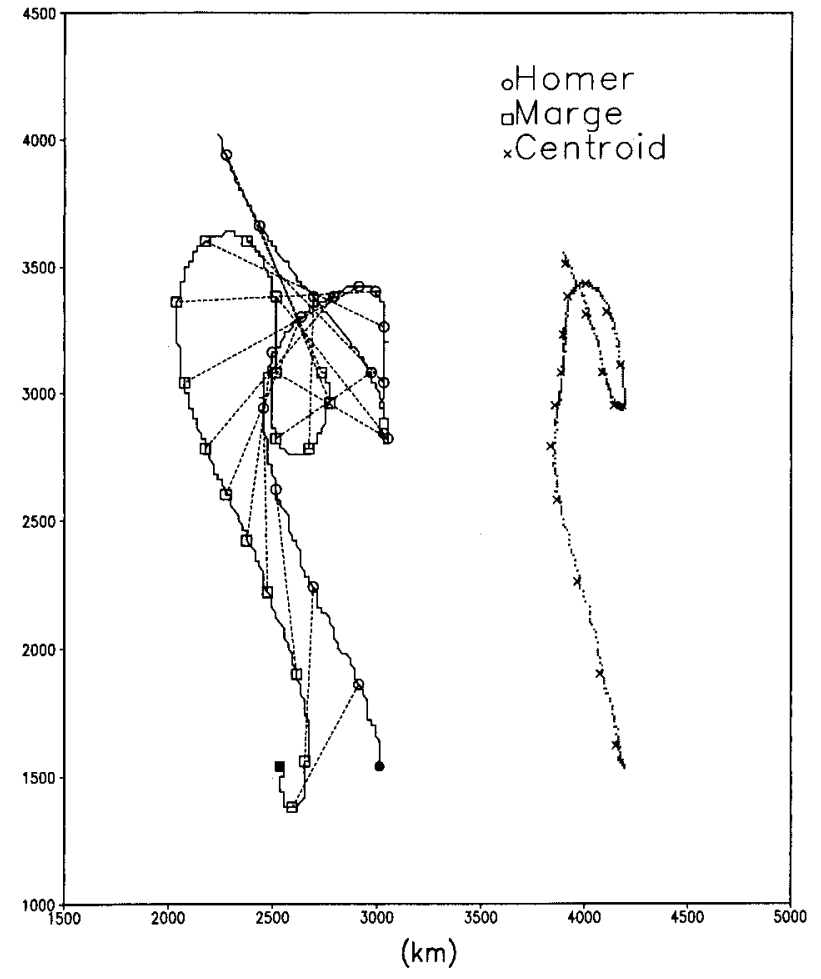

(b)

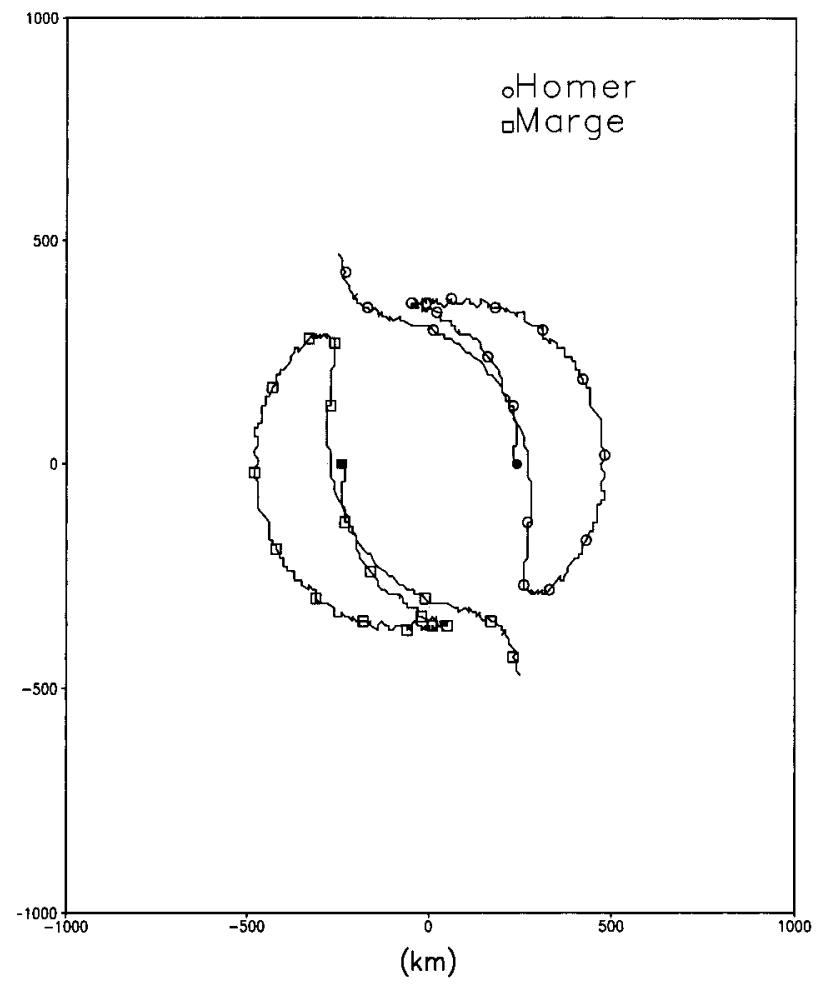

(c)

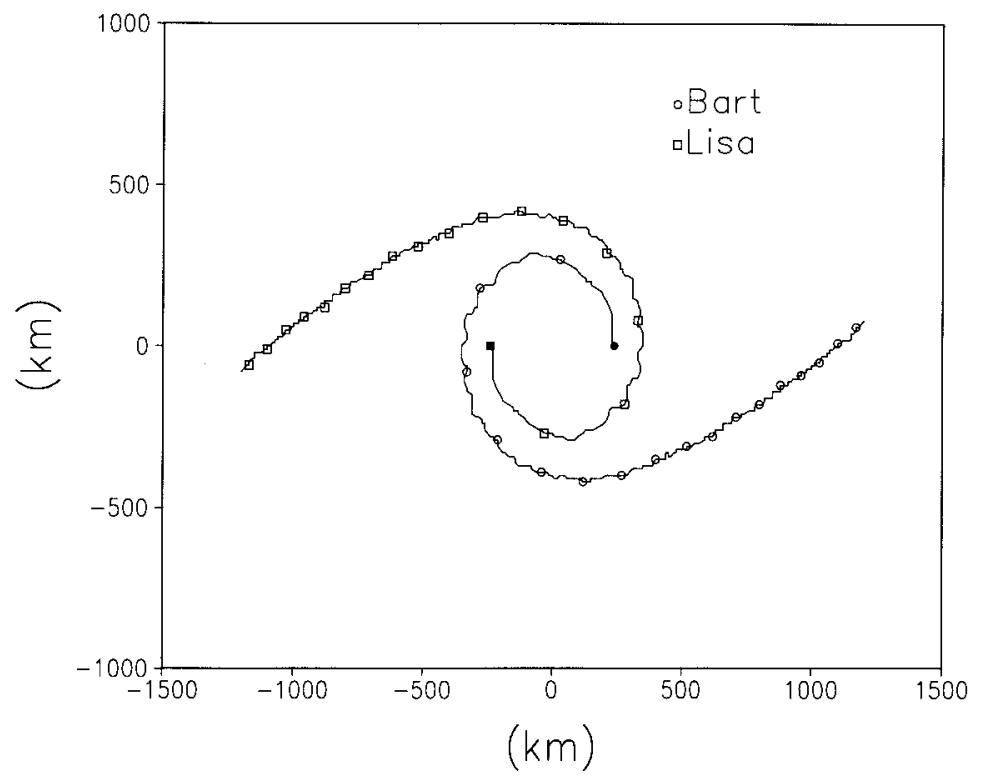

FIG. 5. Vortex tracks for the control experiment, CON2. Vortex interaction is taking place in a quiescent environment on a $\beta$ plane centered at $20^{\circ} \mathrm{N}$. (a) Earth-relative motion, (b) centroid-relative motion, and (c) centroid-relative motion for the same vortices on an $f$ plane. Initial positions are given by the solid circles and squares, with subsequent 24-h positions denoted by open symbols. For visual clarity, the centroid path in (a) has been displaced $1500 \mathrm{~km}$ to the east. Coincident locations of the two vortices are joined by dashed lines to accentuate the relative displacements of the vortices. Note that only a subset of the model domain is shown. 

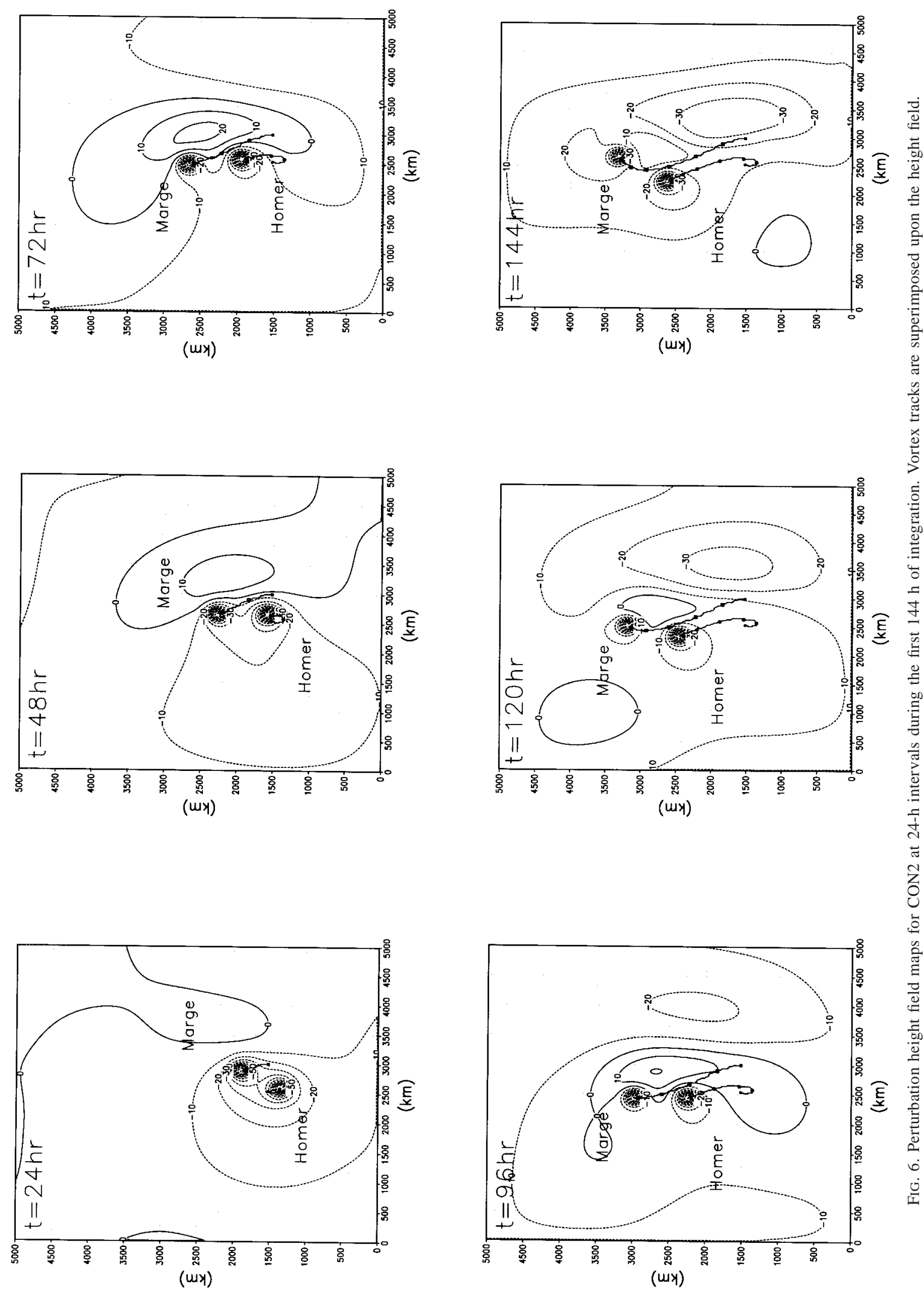

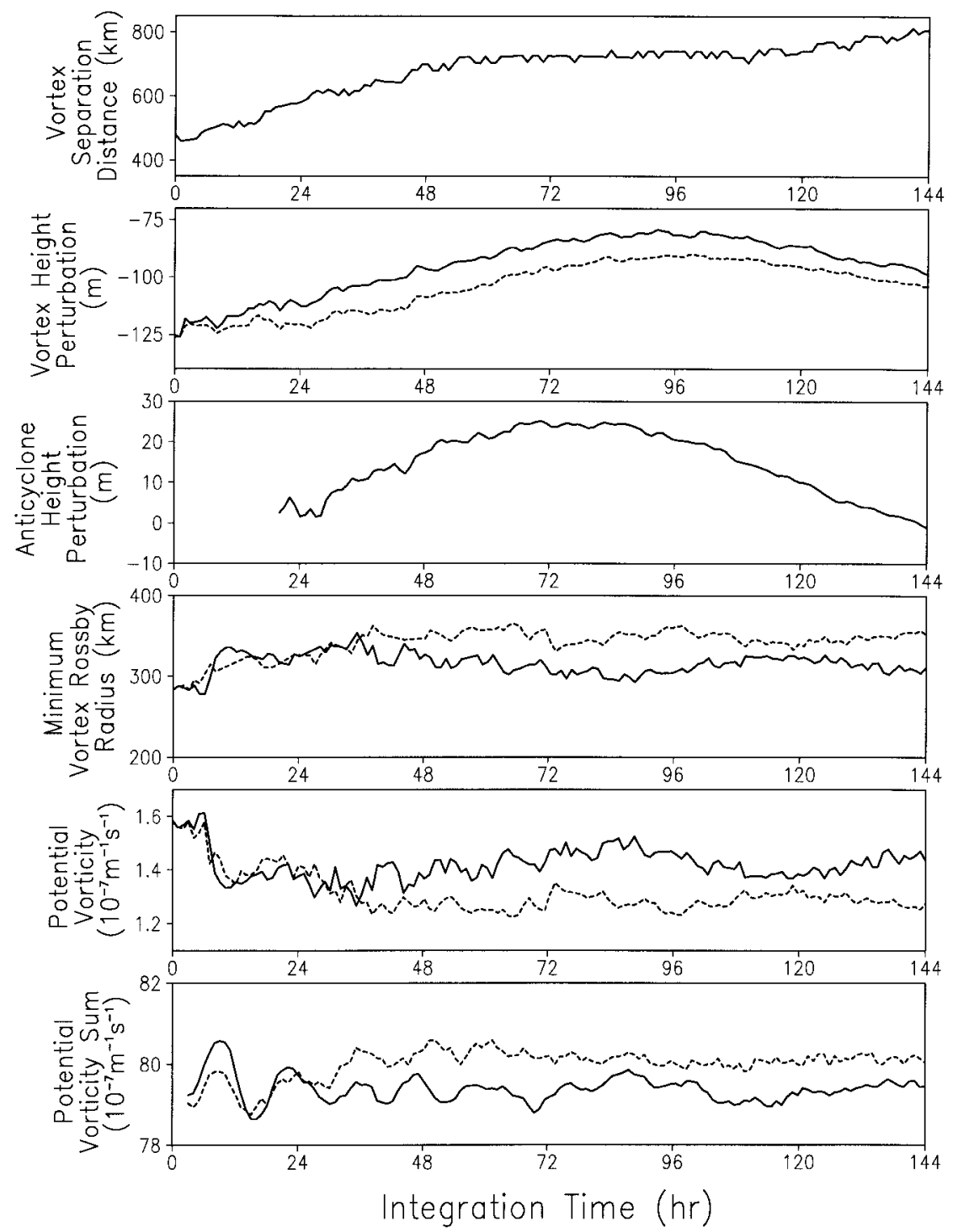

FIG. 7. Time series plots of (a) vortex separation distance $(\mathrm{km})$, (b) vortex height perturbation (m), (c) $\beta$-induced anticyclone height perturbation (m), (d) minimum Rossby deformation radius associated with the vortex $(\mathrm{km})$, (e) maximum PV $\left(\mathrm{m}^{-1} \mathrm{~s}^{-1}\right)$, and (f) areally summed PV $\left(\mathrm{s}^{-1}\right.$ $\mathrm{m}^{-1}$ ) within the radius of maximum winds during $144 \mathrm{~h}$ of integration. A 6-h time-averaging has been applied to (d) to reduce noise due to finite-difference approximations to the integral. Solid and dotted lines represent the vortices "Homer" and "Marge," respectively.

After initialization, the two vortices located in a linear, anticyclonic shear (SLA) move apart, rotating cyclonically for the first $72 \mathrm{~h}$, then anticyclonically, recovering their initial east-west orientation by $144 \mathrm{~h}$ as they cross the ridge axis $(y=2500 \mathrm{~km})$. Once across the ridge axis, the relative cyclonic motion begins again (Fig. 8b). As soon as a significant north-south separation has built up in this region of sheared westerlies, the vortices move apart quickly, largely through differential environmental advection. The tracks for the vortex pair in quadratic, anticyclonic shear (SQA) are very similar to this case (Fig. 8c), even to the timing of the track transitions.
The vortex pair in a cyclonically sheared environment (SLC) exhibit far different motion characteristics to those seen in the other experiments (Fig. 8d). In a centroid-relative sense the vortices are always moving apart here, but this is manifest as a series of cyclonic "hops" (Fig. 8d). The initial location of the vortices is at $y=$ $1600 \mathrm{~km}$, well south of the trough axis at $2500 \mathrm{~km}$. In the first $72 \mathrm{~h}$ the vortices rotate $135^{\circ}$ cyclonically so that the storm initially in the eastern part of the domain is now located farthest west and north of its partner. This is the storm that will move most rapidly away as soon as it transitions across the environmental trough axis. The abrupt relative motion changes indicated in 

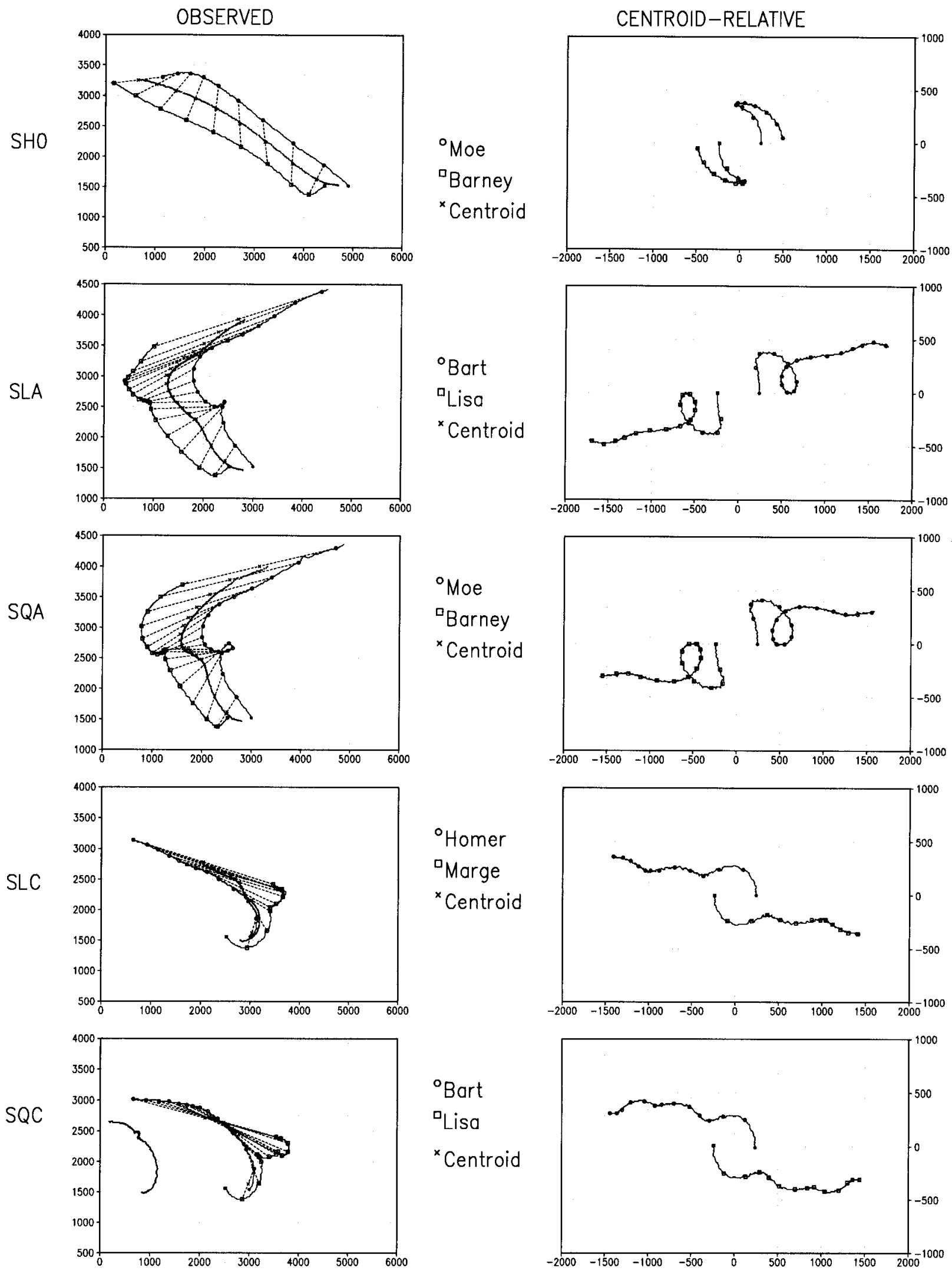

FIG. 8. Observed (left) and centroid-relative (right) vortex tracks for remaining experiments. The vortex interaction is occurring on a $\beta$ plane at $20^{\circ} \mathrm{N}$. 
Fig. 8d correspond to (i) $72 \mathrm{~h}$, when the two vortices have rotated well over $90^{\circ}$ from their initial orientation to a northwest-southeast orientation. The boundary of the northernmost vortex is just intruding into the trough axis; and, (ii) $168 \mathrm{~h}$, the time at which the northernmost vortex has just passed through the trough.

Further evidence of environmental influence on track and propagation can be seen when examining the centroid propagation paths. These are tracks that are attributable to $\beta$ and the environmental vorticity gradient. Figure 10 is a plot of these paths for each of the experiments here. The classic northwest " $\beta$ drift" of the centroid can be seen in CON2 and SH0. Despite the spatially invariant flow associated with $\mathrm{SH} 0$, a $50-\mathrm{km}$ track deviation is observed between CON2 and SH0. This can be explained by the gradient of environmental perturbation height field across the vortices. As the vortex develops asymmetries, nonlinear interaction with the height gradient results, producing a slight track deviation.

In the cyclonically sheared experiments (SLC and SQC), the centroid propagation is deviated to the left (westward) of $\beta$-only (SH0 or CON2). The linear shear (SLC) has a greater deviation than quadratic (SQC), because the local magnitude of the shear at lower latitudes in the domain is greater for the linear profile. In the anticyclonically sheared environments (SLA, SQA), the centroid propagation is deviated to the right (northward) of $\beta$-only. In all shear experiments, however, there is a westward deviation and dramatic slowing of the centroid propagation after $48 \mathrm{~h}$. These centroid propagation deviations of sheared environments from $\beta$-only are in agreement with the results of Wang et al. (1997). Centroid propagation from $48 \mathrm{~h}$ to $96 \mathrm{~h}$ is also slightly faster when the environmental shear enhances the $\beta$-effect (SLC, SQC) than when the environment opposes $\beta$. This confirms Evans et al. (1991), but contradicts the propagation speed patterns found by Wang et al. (1997). This difference might be explained by the significantly differing shear magnitudes between the experiments here and Wang et al. (1997) $\left(0.8 f_{0}\right.$ and $0.12 f_{0}$, respectively).

\section{Links between structure and motion}

As outlined above, one plausible explanation for the track variations observed involves a temporary coupling of the initially distinct vortices into a cooperative system. The propagation tendencies described above for the CON2 experiment are consistent with this idea.

Further evidence for the proposed coupling is found in the PV plots for the SLA (anticyclonic) experiment (Figs. 9a-f), and the SLC (cyclonic) experiment (Figs. $9 g-1)$. The relative orientations of the SLA vortices, across the propagation direction (Figs. 9a-c; 0-48 h), regressing back to east-west (Figs. 9d-f; 72-120 h) as they cross the ridge axis together, are in sharp contrast to the "draughting" of one vortex by the other evident in the SLC run (Figs. 9h-1; 24-120 h). In Figs. 9b-d, filaments of high PV air seem to link the outer regions of the two vortices. This connection appears to be breaking down as the vortices cross the ridge axis (Figs. 9ef).

Placed in a cyclonically sheared flow, the vortex pair in the SLC experiment sustain a northwest-southeast orientation from 72 to $168 \mathrm{~h}$. Over this time, the two vortices appear to have coupled (evident in their propagation speed and their PV signatures; Figs. 9i-1). This link remains unbroken at $168 \mathrm{~h}$, although the vortices are moving apart rapidly at this time due to differential advection.

The impact of vortex coupling beyond $48 \mathrm{~h}$ can be seen in the centroid propagation paths (Fig. 10). Previously it was argued that the vortex coupling would lead to an increase in "effective vortex size," and a decrease in the meridional motion of the centroid. This is shown well in Fig. 10. In all experiments, including $\mathrm{CON} 2$ and $\mathrm{SH}$, an increase in centroid propagation (especially meridional) is shown beyond 48-96 h, during the time when vortex coupling is most evident (Fig. 9).

\section{Conclusions}

The interaction of dual vortices in horizontally sheared environmental flows on a $\beta$ plane has been examined. The study expands upon and synthesizes the work of barotropic single vortex motion in sheared environments (Evans et al. 1991; Williams and Chan 1994; Chan and Law 1995) and barotropic dual-vortex interaction in quiescent environments (Chang 1982, 1984; Ritchie and Holland 1993; Holland and Dietachmayer 1993). The results of this work have illuminated the dynamic life cycle of vortex interaction within environmental shear more clearly.

Vortex interaction becomes evident almost immediately $(<4 \mathrm{~h})$ with the development of vortex Rossby waves. These waves are produced from a PV perturbation that results from: (a) a randomly forced wind perturbation, (b) the presence of a uniform or sheared background flow, or (c) a perturbation by another vortex. During the first 50-75 h of integration, the vortex Rossby waves are merely incidental and do not significantly alter the wind or height profiles. However, as the waves propagate outward at $5 \mathrm{~m} \mathrm{~s}^{-1}$, they approach a critical radius where vortex vorticity changes sign. At this radius, the waves can no longer propagate outward, and the waves' PV filament within the outer region of the vortices. As PV is conserved, the PV filaments are then stretched by the vortex circulation. This forms a band of high PV that surrounds the vortex pair near the radius of vorticity sign reversal. By 72-96 h, this band of high PV has linked the vortices in their outer regions, and the vortices begin exhibiting signs of coupling. The vortex couplet shows an increase in centroid propagation, consistent with previous results showing a relationship 

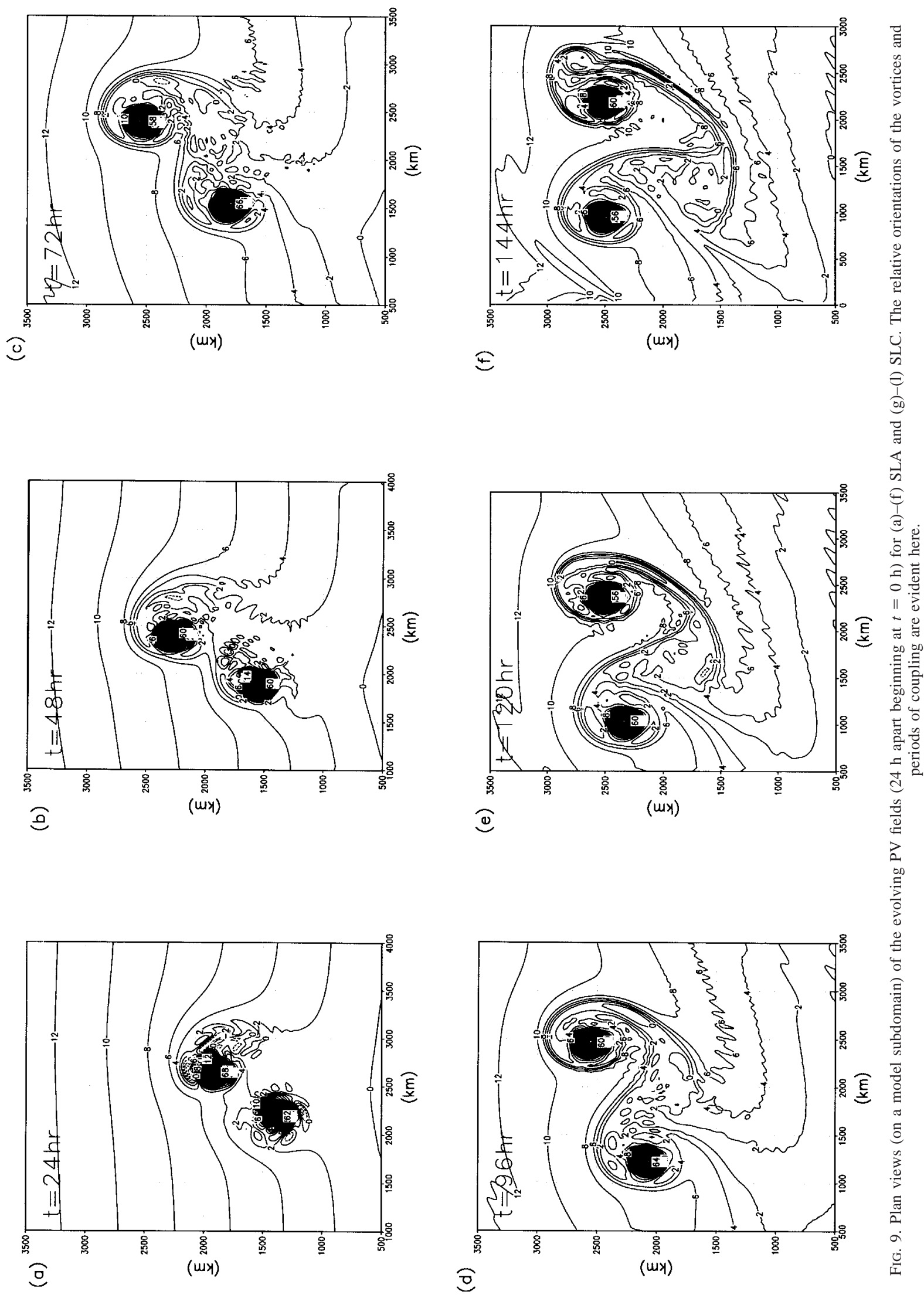

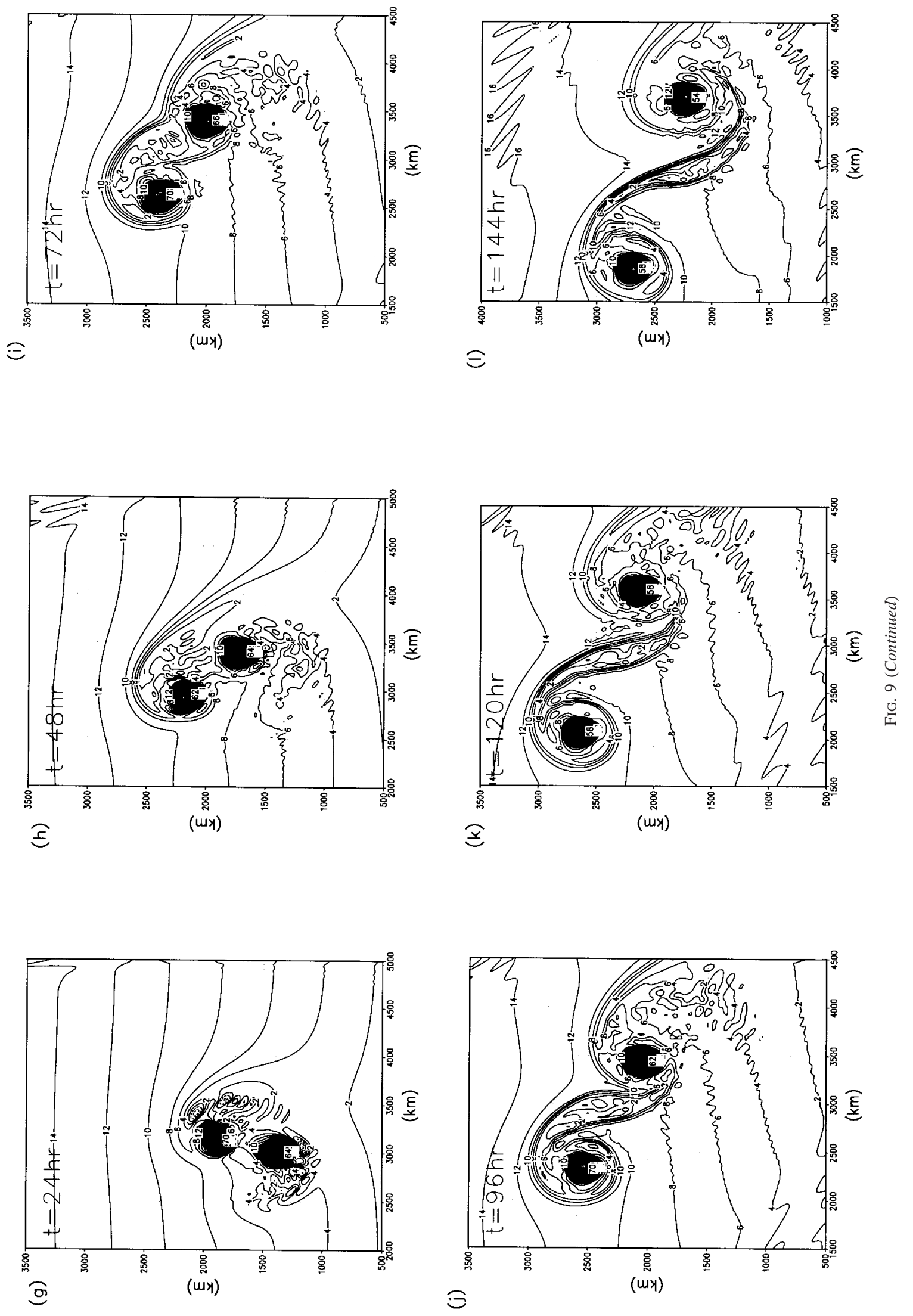


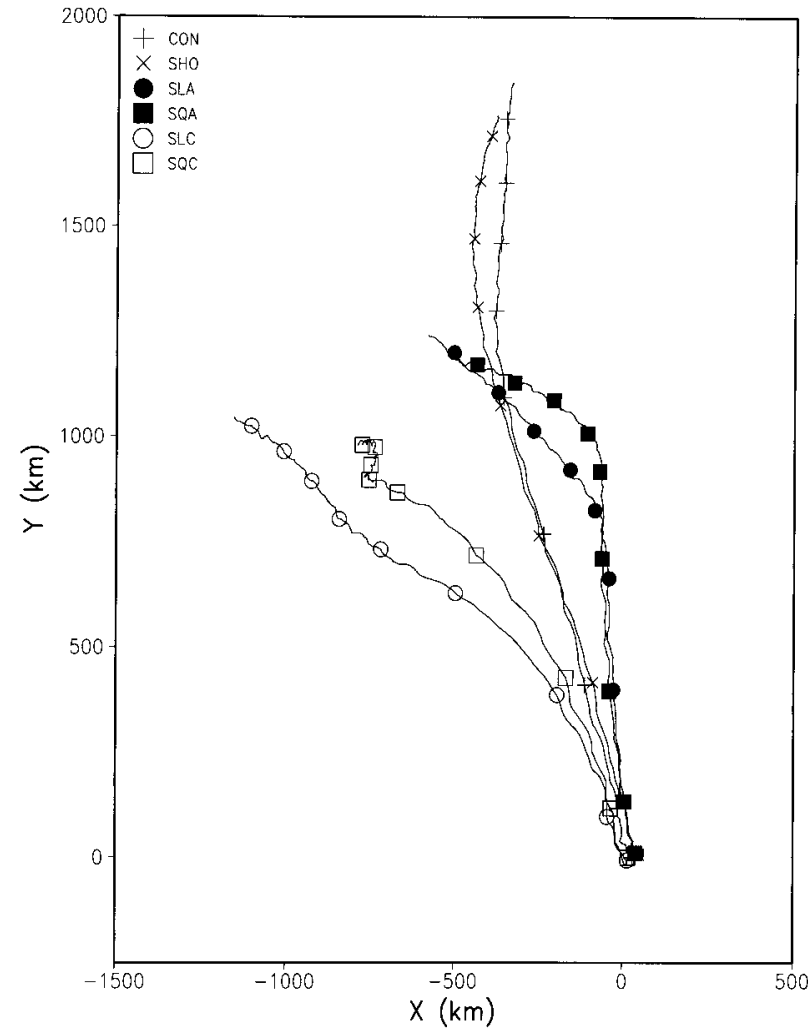

FIG. 10. Comparison of centroid propagation tracks for each case: CON2, SH0, SLC, SQC, SLA, and SQA. The initial centroid location in each case has been reset to $(0,0)$. Note the stronger westward (weaker northward) propagation for the vortex pair centroid in cyclonic shear. By comparison, larger northward propagation is evident for the vortex centroids in the anticyclonic environment cases. Positions every $24 \mathrm{~h}$ are labeled by the symbols in the legend.

between vortex outer structure and propagation speed (Fiorino and Elsberry 1989). Vortex coupling remains evident for at least $96 \mathrm{~h}$.

The tendency for vortex coupling will almost certainly vary with vortex size, strength, and separation, as well as the structure of the environment in which the vortices are embedded. Comparison of experiments SLC and SQC (or SLA and SQA) provides some evidence for this; however, a more comprehensive study of these sensitivities is ongoing. Once the meridional separation distance between the vortices increases sufficiently, the PV link between the vortices is stressed and the link begins to decay.

The sign of the environmental shear has a substantial impact upon the centroid propagation of the vortices. Cyclonically sheared environments produce an enhancement of the $\beta$ drift, and an increased westward motion of the centroid. Anticyclonically sheared environments oppose $\beta$, and result in a decreased centroid westward motion but increased northward motion. These propagation tendencies confirm the results of Wang et al. (1997). A slight increase in centroid propagation speed is found when the environmental vorticity gradient en- hanced $\beta$ (SLC, SQC), consistent with earlier studies (Evans et al. 1991; Smith and Ulrich 1990).

In summary, the motion of an identical vortex pair in a zonally sheared environment on a $\beta$ plane is modulated by (i) the sense and magnitude of the environmental shear and vorticity gradient; (ii) the earth vorticity gradient; and (iii) the development of a link between the two vortices that leads them to move as one system for a portion (3 or more days) of their evolution. This link is the consequence of filamentation of vortex Rossby waves produced from vortex distortion. Features (i) and (ii) of the motion dependence have been observed in previous single-vortex studies (e.g., Evans et al. 1991; Williams and Chan 1994). Lander and Holland (1993) describe cooperative motion (i.e., mutual advection) of observed vortex pairs, while Fiorino and Elsberry (1989) demonstrate an increasing speed and more westward propagation for vortices of increasing size and strength. Specifically, Fiorino and Elsberry (1989) show that outer vortex wind structure (size and strength) relates to propagation speed, while the vortex strength (rate of change of winds outside the radius of maximum winds) modulates the propagation direction. All of these effects (as well as environmental steering) contribute to the ultimate motion patterns observed for the dual-vortex system represented here; however, the relative contributions of each differ over time.

Acknowledgments. The authors are grateful to three anonymous reviewers for their valuable and critical comments.

\section{REFERENCES}

Adem, J., 1956: A series solution for the barotropic vorticity equation and its application in the study of atmospheric vortices. Tellus, 8, 364-372.

Arakawa, A., and V. R. Lamb, 1981: A potential enstrophy and energy conserving scheme for the shallow water equations. Mon. Wea. Rev., 109, 18-36.

Brand, S., 1970: Interaction of binary tropical cyclones of the western North Pacific Ocean. J. Appl. Meteor., 9, 433-441.

Chan, J. C.-L., and R. T. Williams, 1987: Analytical and numerical studies of the beta-effect in tropical cyclone motion. Part I: Zero mean flow. J. Atmos. Sci., 44, 1257-1265.

—, and A. C. K. Law, 1995: The interaction of binary vortices in a barotropic model. Meteor. Atmos. Phys., 56, 135-155.

Chang, S. W., 1982: A numerical study of the interactions between two tropical cyclones. Mon. Wea. Rev., 111, 1806-1817.

—, 1984: Reply. Mon. Wea. Rev., 112, 1646-1647.

DeMaria, M., and J. C. L. Chan, 1984: Comments on "A numerical study of the interactions between two tropical cyclones." Mon. Wea. Rev., 112, 1643-1645.

Dong, K., and C. J. Neumann, 1983: On the relative motion of binary tropical cyclones. Mon. Wea. Rev., 111, 945-953.

Evans, J. L., G. J. Holland, and R. L. Elsberry, 1991: Interactions between a barotropic vortex and an idealized subtropical ridge. Part I: Vortex motion. J. Atmos. Sci., 48, 301-314.

Fiorino, M., and R. L. Elsberry, 1989: Contributions to tropical cyclone motion by small, medium and large scales. Mon. Wea. Rev., 117, 721-727.

Fujiwhara, S., 1921: The natural tendency towards symmetry of mo- 
tion and its application as a principle of motion. Quart. J. Roy. Meteor. Soc., 47, 287-293.

Helmholtz, H., 1867: On integrals of the hydrodynamical equations which express vortex motion. Philos. Mag., 33, 485-512.

Holland, G. J., and G. S. Dietachmayer, 1993: On the interaction of tropical-cyclone-scale vortices. Part III: Continuous barotropic vortices. Quart. J. Roy. Meteor. Soc., 119, 1381-1398.

Kirchoff, G. R., 1876: Vorlesungen über Matematische Physik, Vol. 1. Teubner, $466 \mathrm{pp}$.

Lander, M., and G. J. Holland, 1993: On the interaction of tropicalcyclone-scale vortices. Part I: Observations. Quart. J. Roy. Meteor. Soc., 119, 1347-1361.

Liu, K. S., and J. C. L. Chan, 1997: Sizes of tropical cyclones as inferred from ERS-1 and ERS-2 data. Department of Physics and Materials Science Research Report AP-97-08, City University of Hong Kong, 24 pp. [Available from Department of Physics and Materials Science, City University of Hong Kong, Tat Chee Avenue, Kowloon, Hong Kong.]

Montgomery, M. T., and R. J. Kallenbach, 1997: A theory for vortex
Rossby-waves and its application to spiral bands and intensity changes in hurricanes. Quart. J. Roy. Meteor. Soc., 123, 435465.

Ritchie, E. A., and G. J. Holland, 1993: On the interaction of tropicalcyclone-scale vortices. Part II: Discrete vortex patches. Quart. J. Roy. Meteor. Soc., 119, 1363-1379.

Satomura, T., 1980: Dynamic normal mode initialization for a limited area model. J. Meteor. Soc. Japan, 66, 261-276.

Shapiro, L. J., and M. T. Montgomery, 1993: A three-dimensional balance theory for rapidly rotating vortices. J. Atmos. Sci., 50, 3322-3335.

Smith, R. K., and W. Ulrich, 1990: An analytic theory of tropical cyclone motion using a barotropic model. J. Atmos. Sci., 47, 1973-1986.

Wang, B., X. Li, and L. Wu, 1997: Direction of hurricane beta drift in horizontally sheared flows. J. Atmos. Sci., 54, 1462-1471.

Williams, R. T., and J. C. L. Chan, 1994: Numerical studies of the beta effect in tropical cyclone motion. Part II: Zonal mean flow experiments. J. Atmos. Sci., 51, 1065-1076. 